\title{
Identification of Prognostic Biomarkers in the Urinary Peptidome of the Small Renal Mass
}

Ashley Di Meo, ${ }^{* \dagger \ddagger}$ Ihor Batruch, ${ }^{\S}$ Marshall D. Brown, ${ }^{\llbracket}$ Chuance Yang, ${ }^{\|}$Antonio Finelli, ${ }^{* *}$ Michael A.S. Jewett, ** Eleftherios P. Diamandis, ${ }^{\ddagger 8}$ and George M. Yousef ${ }^{* \dagger \neq}$

From the Department of Pediatric Laboratory Medicine, ${ }^{*}$ Hospital for Sick Children, Toronto, Ontario, Canada; the Department of Laboratory Medicine, ${ }^{\dagger}$ Li Ka Shing Knowledge Institute, St. Michael’s Hospital, Toronto, Ontario, Canada; the Department of Laboratory Medicine and Pathobiology, ${ }^{\ddagger}$ University of Toronto, Toronto, Ontario, Canada; the Department of Pathology and Laboratory Medicine, ${ }^{\S}$ Mount Sinai Hospital, Toronto, Ontario, Canada; the Department of Biostatistics, "Fred Hutchinson Cancer Research Center, Seattle, Washington; the Department of Urology," The Second Affiliated Hospital of Xi'an Jiaotong University, Xi'an, China; and the Department of Surgical Oncology, ** Princess Margaret Cancer Centre, University Health Network, Toronto, Ontario, Canada

Accepted for publication August 20, 2019.

Address correspondence to George M. Yousef, M.D., Ph.D., F.R.C.P.C. (Path), Department of Pediatric Laboratory Medicine, The Hospital for Sick Children, 555 University Ave., Toronto, ON, M5G 1X8, Canada. E-mail: george. yousef@sickkids.ca.

\begin{abstract}
Renal cell carcinoma (RCC) is often diagnosed incidentally as a small renal mass (SRM; pT1a, $\leq 4 \mathrm{~cm}$ ). Increasing concerns surrounding the overtreatment of patients with benign or clinically silent SRMs has resulted in a recent shift in treatment recommendations, especially in elderly and infirm patients. There are currently no biomarkers that can predict progression. We used a quantitative label-free liquid chromatography-tandem mass spectrometry peptidomics approach and targeted parallel-reaction monitoring to identify early, noninvasive diagnostic and prognostic biomarkers for early-stage RCCSRMs. In total, 115 urine samples, including 33 renal oncocytoma $(\leq 4 \mathrm{~cm})$ cases, 30 progressive and 26 nonprogressive clear cell RCC-SRM cases, and 26 healthy controls were evaluated. Nine endogenous peptides that displayed significantly elevated expression in clear cell RCC-SRMs relative to healthy controls were identified. Peptides NVINGGSHAGNKLAMQEF, VNVDEVGGEALGRL, and VVAGVANALAHKYH showed significantly elevated expression in clear cell RCC-SRMs relative to renal oncocytoma. Additionally, peptides SHTSDSDVPSGVTEVVVKL and IVDNNILFLGKVNRP displayed significantly elevated expression in progressive relative to nonprogressive clear cell RCC-SRMs. Peptide SHTSDSDVPSGVTEVVVKL showed the most significant discriminatory utility (area under the curve, 0.76; 95\% CI, 0.62-0.90; $P=0.0027$ ). Patients with elevated SHTSDSDVPSGVTEVVVKL expression had significantly shorter overall survival (hazard ratio, 4.13; 95\% CI, 1.09-15.65; $P=0.024$ ) compared to patients with low expression. Pretreatment characterization of urinary peptides can provide insight into early RCC progression and may aid clinical decision-making and improve disease management. (Am J Pathol 2019, 189: 2366-2376; https://doi.org/10.1016/j.ajpath.2019.08.015)
\end{abstract}

Renal cell carcinoma (RCC) is the most fatal urologic malignancy, with a 5 -year survival rate of $<10 \%$ for patients with metastatic disease. ${ }^{1}$ One of the most frequently diagnosed neoplasms of the genitourinary tract, RCC accounts for approximately $90 \%$ of all renal malignancies. ${ }^{2}$ RCC represents a heterogenous group of tumors with varying clinical outcomes. The most prevalent subtype is clear cell (cc)-RCC, which composes between $70 \%$ to $75 \%$ of all renal tumors. ${ }^{3}$ With relatively nonspecific or absent symptoms, RCC is frequently detected incidentally as a small renal mass (SRM; $\leq 4 \mathrm{~cm}$ ).

SRMs are defined as solid enhancing tumors $\leq 4 \mathrm{~cm}$ in diameter, with image characteristics consistent with a stage
pT1a tumor. ${ }^{4}$ Approximately $30 \%$ of incidentally detected SRMs are benign. ${ }^{5}$ Moreover, RCC-SRMs can be clinically progressive (grow rapidly and metastasize) or nonprogressive. The former requires immediate treatment, whereas the latter, having relatively indolent clinical behavior, can be managed conservatively. The standard treatment of

\footnotetext{
Supported by Canadian Institutes of Health Research grant MOP 119606 (G.M.Y.), Kidney Foundation of Canada grant KFOC130030 (G.M.Y.), and the Kidney Cancer Research Network of Canada and the Canadian Urological Oncology Group research trainee award (A.D.M.).

Disclosures: None declared.
} 
localized RCC is radical nephrectomy. However, the observation that most SRMs have slow growth rates and low malignant potential ${ }^{6}$ has shifted treatment recommendations, especially in elderly patients, in whom the risk of treatment is high. ${ }^{7}$ The low malignant potential of SRMs was recently was recently highlighted in a multicenter Phase II clinical trial of initial active surveillance of SRMs with treatment delayed until progression. The study revealed that local progression rates are low, and that delayed treatment is unlikely to affect long-term outcomes. ${ }^{8}$ However, it remains unclear which tumors are safe to observe, and which require immediate intervention. Tumor size is a poor predictor of pathology, and current radiographic techniques cannot accurately distinguish benign from malignant masses. Furthermore, SRM tissue obtained through renal biopsy is often limited. ${ }^{9}$ The identification of noninvasive biomarkers predicting aggressive behavior could help to guide treatment decisions.

Liquid biopsy has emerged as a tool useful for cancer biomarker discovery and represents a noninvasive and costeffective alternative to tissue biopsy. ${ }^{10}$ Owing to its minimally invasive nature, liquid biopsy can be scheduled more frequently, providing a more personalized snapshot of disease. In addition, liquid biopsy can be used over periods of active surveillance to monitor disease progression. It may also better reflect tumor heterogeneity by capturing the genetic profile of all tumor subclones. ${ }^{9}$ Urine is routinely used as a liquid biopsy for clinical diagnosis as it is easily accessible. Urine is also a biological matrix ideal for kidneyrelated diagnostics because it contains analytes originating from the kidneys. ${ }^{11,12}$

Proteomic analysis has led to the discovery of clinically relevant proteins. Peptidomic analysis adds another dimension to proteomic analysis by enabling the identification of native or endogenous peptides derived from the aberrant activity of specific proteases. ${ }^{13}$ Several reports have demonstrated the utility of naturally occurring peptides in diagnosing RCC. One study identified a panel of 12 urinary peptides that could be used for differentiating RCC from benign lesions and healthy controls. ${ }^{2}$ The same group also identified urinary peptides whose expression varied according to tumor size, stage, and grade in RCC. ${ }^{14}$ Using high-resolution capillary electrophoresis coupled to mass spectrometry, Frantzi et al $^{7}$ identified a panel of 86 urinary peptides that enabled the detection of RCC. Moreover, Huang et $\mathrm{al}^{15}$ developed a highly predictive model that could be used for classifying RCC versus healthy controls.

This study aimed to identify early, noninvasive biomarkers of clinically localized RCC. Urine specimens from patients with ccRCC-SRM (pT1a; $\leq 4 \mathrm{~cm})$ or renal oncocytoma $(\leq 4 \mathrm{~cm})$ and healthy controls were analyzed using label-free liquid chromatography-tandem mass spectrometry (LC-MS/MS) and targeted parallel-reaction monitoring (PRM). Urinary peptides that could be used for distinguishing ccRCC-SRMs from healthy controls and renal oncocytoma $(\leq 4 \mathrm{~cm})$ were identified. Moreover, two urinary peptides that could be used for distinguishing progressive from nonprogressive ccRCC-SRMs were identified.

\section{Materials and Methods}

\section{Specimen Collection}

The study protocol was approved by the Research Ethics Board of St. Michael's Hospital and the University Health Network (Toronto, Ontario, Canada). Informed consent was obtained from all participants before study enrollment. Urine samples were collected from patients enrolled in a multicenter, prospective, Phase II clinical trial of active surveillance with treatment delayed until progression, from 2004 to $2009 .{ }^{8}$ Serial imaging was performed with computed tomography, magnetic resonance imaging, or ultrasound at months 3 and 6 , then every 6 months until 3 years, and then each year. All patients enrolled in the study were asked to undergo percutaneous needle core biopsy for pathologic diagnosis. Diagnosis was confirmed and reviewed by a pathologist according to the 2002 TMN System and the 2004 World Health Organization classification. Urine samples were collected before percutaneous needle core biopsy. Using linear mixed-effects analysis, ccRCC-SRMs were classified as progressive or nonprogressive (Supplemental Figure S1). Patients with local progression, which was defined as: i) SRM growth of $\geq 4 \mathrm{~cm}$ in diameter, ii) a doubling of calculated SRM volume in $\leq 12$ months, or iii) metastasis, were treated by partial or radical nephrectomy. In patients treated for local progression, diagnosis was confirmed on resected tumor tissues. Samples were collected from Princess Margaret Cancer Center, Toronto, Canada. For the discovery phase, 24 urine samples were collected (Supplemental Table S1), including six oncocytoma $(\leq 4 \mathrm{~cm})$ cases, seven progressive and five nonprogressive ccRCC-SRM cases, and six healthy controls. For the validation phase, an independent set of 91 urine samples was collected (Supplemental Table S1), including 27 oncocytoma $(\leq 4 \mathrm{~cm})$ cases, 23 progressive and 21 nonprogressive ccRCC-SRM cases, and 20 healthy controls. All participants enrolled in the study had no history of kidney-related or systemic disease.

\section{Sample Preparation for Label-Free Quantification}

Urine samples were thawed on ice and centrifuged at 2000 $\times g$ for 10 minutes. Urine volumes were normalized to 90 $\mu$ mol creatinine per sample (approximately 3 to $10 \mathrm{~mL}$ per individual). Individual samples were adjusted to $\mathrm{pH} 8.0$ by the addition of ammonium bicarbonate and concentrated using Vivaspin 20-mL, 10-kDa cutoff membranes (Sartorius Stedim Biotech, Göttingen, Germany), according to the manufacturer's protocol. Individual samples were reduced with $2 \mathrm{mmol} / \mathrm{L}$ dithiothreitol (Sigma-Aldrich, 
Oakville, Canada) at room temperature for 30 minutes, then alkylated with $4 \mathrm{mmol} / \mathrm{L}$ iodoacetamide (SigmaAldrich) at room temperature for 45 minutes in the dark. Each sample was acidified to $\mathrm{pH} 4.0$ by the addition of formic acid. Samples were passed through Oasis HLB cartridges [1 mL (30 mg); Waters, Milford, MA], as described previously, ${ }^{12}$ and mixed with equal volumes of ethyl acetate. Samples were then centrifuged at 17,000 $\times g$ for 5 minutes. After the removal of the upper layer, samples were reduced to $200 \mu \mathrm{L}$ using the Speedvac system.

\section{Strong Cation Exchange Chromatography}

Each sample was diluted 1:2 in mobile phase A SCX buffer (0.26 M FA, 5\% ACN; pH 2 to 3) and loaded directly onto a $500-\mu \mathrm{L}$ loop connected to a PolySufloethyl A column $(2.1 \mathrm{~mm} \times 200 \mathrm{~mm} ; 5 \mu \mathrm{m} ; 200 \AA$; The Nest Group Inc., Southborough, MA). Fractionation was performed using an Agilent 1100 HPLC system (Agilent Technologies, Santa Clara, CA). Peptides were eluted at a flow rate of $200 \mu \mathrm{L} /$ minute over a 60 -minute gradient consisting of mobile phase A and mobile phase B SCX buffers $(0.26 \mathrm{M}$ formic acid, 5\% acetonitrile, $1 \mathrm{M}$ ammonium formate; $\mathrm{pH} 4$ to 5). Sample fractionation was monitored at a wavelength of 280 $\mathrm{nm}$. Fractions were collected every 2 minutes from 10 to 55 minutes, and those with low peak absorbance were pooled, resulting in a total of 72 SCX fractions per sample.

\section{Peptide Identification by LC-MS/MS}

Individual samples were concentrated using C-18 Bond Elut OMIX Tips (Agilent Technologies), eluted in $5 \mu \mathrm{L}$ of $65 \%$ buffer B ( $0.1 \%$ formic acid in acetonitrile) and $35 \%$ buffer A $(0.1 \%$ formic acid in water), and finally diluted with 60 $\mu \mathrm{L}$ of buffer A. Each sample was loaded $(18 \mu \mathrm{L})$ onto a 3.2 $\mathrm{cm} \mathrm{C}_{18}$ trap column $\left(5-\mu \mathrm{m} \mathrm{C}_{18}\right.$ particle, $150-\mu \mathrm{m}$ inner diameter) using the Easy-nLC 1000 system (Thermo Fisher Scientific, San Jose, CA) running buffer A. Peptides were eluted onto a resolving 15-cm-long PicoTip Emitter $(3-\mu \mathrm{m}$ $\mathrm{C}_{18}$ particle) with an inner diameter of $75 \mu \mathrm{m}(8-\mu \mathrm{m}$ tip; New Objective, Woburn, MA) at a flow rate of $300 \mathrm{~nL} /$ minute. The LC setup was coupled online to a Q-Exactive (Thermo Fisher Scientific) mass spectrometer with a nanoelectrospray ionization source. A 60-minute datadependent acquisition method was set up on the Q-Exactive (Thermo Fisher Scientific) mass spectrometer. The full MS1 scan from $\mathrm{m} / \mathrm{z} 400$ to 2000 was acquired in the Orbitrap at a resolution of 70,000. Automatic gain control for MS1 was set to $3 \times 10^{6}$ with a maximum injection time of 100 milliseconds. Automatic gain control target for MS2 was set to $5 \times 10^{4}$ with a maximum injection time of $50 \mathrm{~ms}$, and normalized collision energy (NCE) of 27. Samples were analyzed in duplicate, and reproducibility was confirmed by running $0.1 \mathrm{fmol} / \mu \mathrm{L}$ bovine serum albumin, every 10 runs.

\section{Data Analysis and Label-Free Quantification}

XCalibur software version 2.0.6 (Thermo Fisher Scientific) was used to generate raw files. Raw files were uploaded into MaxQuant version 1.5.2.8 ${ }^{16}$ (http://coxdocs.org/doku.php? $i d=$ maxquant:viewer:start) for label-free quantification. MaxQuant searches were performed against the nonredundant SwissProt Human Protein database (HUMAN_sProt01072015). Search parameters included unspecific enzyme digestion, fixed carbamidomethylation of cysteines, and variable methionine and proline oxidation. A maximum of 50 missed cleavages was allowed. Raw files were initially searched against a human-first-search database with a parent tolerance of $20 \mathrm{ppm}$ to calculate and adjust the parent tolerance to $4.5 \mathrm{ppm}$ for the search against the SwissProt Human Protein database. The SwissProt Human Protein database was randomized and the false detection rate was set to $1 \%$. Data were analyzed using label-free quantification, and the match-between-runs interval was set to 2 minutes. ${ }^{17}$

\section{Selection of Peptides and PRM Method Development}

Peptide selection was based on specific criteria. Peptides that displayed significantly elevated expression in cases relative to controls were preferentially selected. Peptides were also ranked based on biomarker performance. To identify peptides likely to validate, 71 unscheduled survey PRM assays were developed. Pooled unfractionated urine specimens were run on the Q-Exactive Plus mass spectrometer (Thermo Fisher Scientific), and precursor and fragment ions were viewed in XCalibur version 2.0.6 and Skyline software version 4.2 (MacCoss Lab Software, Seattle, WA). In total, 40 peptides were selected for PRM method development. Peptide uniqueness was verified using the Basic Local Alignment Search Tool version 4 (National Center for Biotechnology Information, Bethesda, MD; http://blast.ncbi.nlm.nih.gov/Blast.cgi, last accessed April 30, 2018).

We experimentally verified the presence of candidate peptides using crude heavy-labeled peptides. In total, 40 heavy labeled peptides (JPT Peptide Technology, Berlin, Germany) were individually reconstituted in $130 \mu \mathrm{L}$ of $20 \%$ acetonitrile in $0.1 \mathrm{M}$ ammonium bicarbonate and stored at $-20^{\circ} \mathrm{C}$. In the first step of method development, retention times were verified using the elution profile of heavylabeled peptides in pooled unfractionated urine samples. Eight heavy-labeled peptides could not be identified in the urine matrix. As such, monitoring of these peptides was abolished. In the second step of method development, NCE was optimized. Briefly, a range of NCE values were surveyed for each peptide on the Q-Exactive Plus (Thermo Fisher Scientific). The experimentally observed retention time and optimized NCE values were then used to develop a single multiplexed, scheduled PRM method. In the third step, calibration curves were generated, by spiking heavylabeled peptides into pooled unfractionated urine samples at 
concentrations of 10,000,1000,100,10,1, 0.1, and $0 \mathrm{fmol} /$ $\mu \mathrm{L}$. Samples were analyzed in triplicate and run on the QExactive Plus (Thermo Fisher Scientific). The lower limit of detection and quantification of each peptide were calculated. The limit of detection was defined as the lowest analyte concentration that could be distinguished from the background $(S / N \geq 3)$. The limit of quantification was defined as the lowest analyte concentration measured ( $\mathrm{CV} \leq 20 \%)$ within the linear range of the calibration curve. Heavylabeled peptide amount was optimized so that the ratio of light and heavy peptide was close to 1 . Overall, 32 endogenous peptides and 146 transitions were scheduled in a single multiplexed, scheduled PRM method with optimized NCE values (Supplemental Table S2). Retention time windows were set at \pm 3.5 minutes from the experimentally verified elution times and were optimized to ensure the acquisition of at least 8 to 10 points per peak.

\section{PRM Sample Processing}

A targeted, multilevel profiling approach was developed (Supplemental Figure S2). Individual samples were centrifuged at $2000 \times g$ for 10 minutes, adjusted to $\mathrm{pH} 8.0$ by the addition of ammonium bicarbonate, and concentrated using Vivaspin $2 \mathrm{~mL} 10 \mathrm{kDa}$ cutoff membranes (Sartorius Stedim Biotech), according to the manufacturer's protocol. Filtrate samples were separated into two $500-\mu \mathrm{L}$ aliquots and processed in parallel. Heavy-labeled peptides (JPT Peptide Technologies) were spiked into each sample. Individual samples were then reduced with $2 \mathrm{mmol} / \mathrm{L}$ dithiothreitol, followed by alkylation with $4 \mathrm{mmol} / \mathrm{L}$ iodoacetamide, as described in Sample Preparation for Label-Free Quantification. Each sample was acidified to $\mathrm{pH} 4.0$ by the addition of formic acid. Samples were passed through OASIS HLB cartridges [1 mL $(30 \mathrm{mg})$, Waters], as described previously, ${ }^{12}$ and mixed with an equal volume of ethyl acetate. Samples were then centrifuged at $17,000 \times g$ for 5 minutes. After the removal of the upper layer, samples were reduced to $200 \mu \mathrm{L}$ using the Speedvac system.

\section{PRM Methods}

Individual samples were concentrated using C-18 Bond Elut OMIX Tips (Agilent Technologies), eluted in $5 \mu \mathrm{L}$ of $65 \%$ buffer B (0.1\% formic acid in acetonitrile) and $35 \%$ buffer A $(0.1 \%$ formic acid in water), and finally diluted in $60 \mu \mathrm{L}$ of buffer A. Each sample was loaded $(18 \mu \mathrm{L})$ onto a 3.2-cm $\mathrm{C}_{18}$ trap column (5 $\mu \mathrm{m} \mathrm{C}_{18}$ particle, $150 \mu \mathrm{m}$ inner diameter) using the EASY-nLC 1000 system (Thermo Fisher Scientific) running buffer $\mathrm{A}$. Peptides were eluted onto a resolving 15-cm-long PicoTip Emitter $\left(3 \mu \mathrm{m} \mathrm{C}_{18}\right.$ particle) with an inner diameter of $75 \mu \mathrm{m}$ ( $8 \mu \mathrm{m}$ tip, New Objective) at a flow rate of $300 \mathrm{~nL} /$ minute. The LC setup was coupled online to a Q-Exactive Plus mass spectrometer (Thermo Fisher Scientific) with a nanoelectrospray ionization source. A 60-minute data-dependent acquisition method was set up on the Q-Exactive Plus mass spectrometer (Thermo Fisher
Scientific). The full MS1 scan from $\mathrm{m} / \mathrm{z} 400$ to 2000 was acquired in the Orbitrap at a resolution of 70,000. Automatic gain control for MS1 was set to $1 \times 10^{6}$ with a maximum injection time of 120 milliseconds. Automatic gain control target for MS2 was set to $2 \times 10^{5}$ with a maximum injection time of $130 \mathrm{~ms}$, and optimized NCE. Samples were analyzed in duplicate and reproducibility was confirmed by running $0.1 \mathrm{fmol} / \mu \mathrm{L}$ bovine serum albumin, every 10 runs.

\section{Data Analysis and Relative Peptide Quantification}

XCalibur software version 2.0.6 was used to generate raw files. Raw files were uploaded into Skyline version 4.2. Skyline was used to identify and visualize transitions, as well as to verify heavy and endogenous peptide coelution. In addition, Skyline was used for peak integration and quantification of the area under the curve (AUC) as well as the light to heavy peptide ratios $\left(\mathrm{AUC}_{\text {light }} / \mathrm{AUC}_{\text {heavy }}\right)$. The average $\mathrm{AUC}_{\text {light }} / \mathrm{AUC}_{\text {heavy }}$ was multiplied by the amount of spiked heavy-labeled peptide to calculate the relative amount of endogenous light peptide. For peptides containing methionine, $\mathrm{AUC}_{\text {light }} / \mathrm{AUC}_{\text {heavy }}$ was calculated as described previously. ${ }^{18}$ Relative light peptide amounts were normalized to creatinine amount as indicated: $\mathrm{AUC}_{\text {light }} / \mathrm{AUC}_{\text {heavy }} \times$ spiked heavy peptide concentration $(\mathrm{fmol})_{\text {average }} /$ urine creatinine concentration $(\mu \mathrm{mol})$.

\section{Clinical Validation on the Cancer Genome Atlas Data Set}

RNA sequencing median $z$-score values and clinical variables were compiled from 195 early-stage (T1) ccRCC patients from The Cancer Genome Atlas (www.cancergenome.nih. gov, last accessed January 18, 2019). Overall survival was assessed in relation to mRNA expression using Cutoff Finder.

\section{Data Availability}

Mass spectrometry raw data files and MaxQuant files were deposited to the ProteomeXchange Consortium via the PRIDE partner repository (https://www.ebi.ac.uk/pridel archive; accession number PXD013111).

PRM data were deposited to the Peptide Atlas repository (http://www.peptideatlas.org, last accessed, August 2, 2019) with the data set identifier PASS01350.

\section{Statistical Analysis}

Statistical analysis was performed using R statistical software version 3.6.1 (www.rproject.org) ${ }^{19}$ and GraphPad Prism version 8.0 (GraphPad Software, San Diego, CA). For the discovery phase, the peptides.txt file was filtered to remove contaminants and reverse identifications. Statistical significance was calculated using the $U$-test. $P$ values of $<0.05$ were considered significant. Volcano plots were generated using Perseus version 1.5.2.6. ${ }^{20}$ Unsupervised hierarchical clustering 
(CLU; Spearman rank) and principal component analysis were performed using GenePattern version 19.09 (https://cloud. genepattern.org, last accessed January 2019). ${ }^{21}$

For the validation phase, normalized expression values were $\log 2$ transformed. Statistically significant differences were determined using the Welch $t$-test. $P$ values of $<0.05$ were considered significant. Receiver operating characteristic analysis was applied and the AUC was calculated to assess performance. Kaplan-Meier curves were constructed using Cutoff Finder (http://molpath.charite.de/cutoff, last accessed January 20,2019), and differences were evaluated by the logrank test $(P<0.05)$. Peptide expression was converted to a dichotomous variable using Cutoff Finder. Associations between peptide status and clinicopathologic variables were determined using the Fisher exact test or Pearson $\chi^{2}$ test.

\section{Results}

\section{Delineating the Urinary Peptidome of Early-Stage ccRCC}

The LC-MS/MS analysis of urine led to the identification and quantification of 20,760 unique endogenous peptides arising from 1418 proteins (Figure 1A). In total, 1091 peptides were differentially expressed between healthy controls and earlystage ccRCC $(P<0.05, U$-test $)$. Of these, 811 peptides were significantly up-regulated, and 280 peptides were significantly down-regulated in early-stage ccRCC (Supplemental Figure S3A). Differentially expressed urinary peptides could be used for discriminating early-stage clear cell tumors from healthy controls using principal component analysis (Supplemental Figure S3B). Unsupervised hierarchical CLU showed that patients from each group could be clustered together based on the expression of urinary peptides (Supplemental Figure S3C).

In addition, 964 peptides were differentially expressed between renal oncocytoma $(\leq 4 \mathrm{~cm})$ and early-stage ccRCC patients. A total of 391 peptides were significantly upregulated, and 573 peptides were significantly downregulated, in ccRCC (Supplemental Figure S4A). Principal component analysis showed that, with one exception, differentially expressed peptides could be used for distinguishing early-stage clear cell tumors from renal oncocytoma $(\leq 4 \mathrm{~cm})$ (Supplemental Figure S4B). Unsupervised hierarchical CLU analysis also showed that patients of each histologic type could be clustered together based on the expression of urinary peptides (Supplemental Figure S4C).

Moreover, 352 endogenous peptides displayed differential expression between progressive and nonprogressive early-stage ccRCC patients. Sixty-nine peptides were significantly up-regulated, and 283 peptides were significantly down-regulated in progressive clear cell tumors (Figure 1B). Differentially expressed peptides could be used for distinguishing progressive from nonprogressive clear cell tumors using principal component analysis (Figure 1C). Unsupervised hierarchical CLU analysis showed that patients from each group could be clustered together based on the expression of urinary peptides (Figure 1D).

\section{Use of Endogenous Peptides in Distinguishing Healthy} Controls from Early-Stage ccRCC

A targeted PRM assay was developed to verify the diagnostic utility of selected candidate peptides identified in the discovery phase. Univariate analysis identified nine
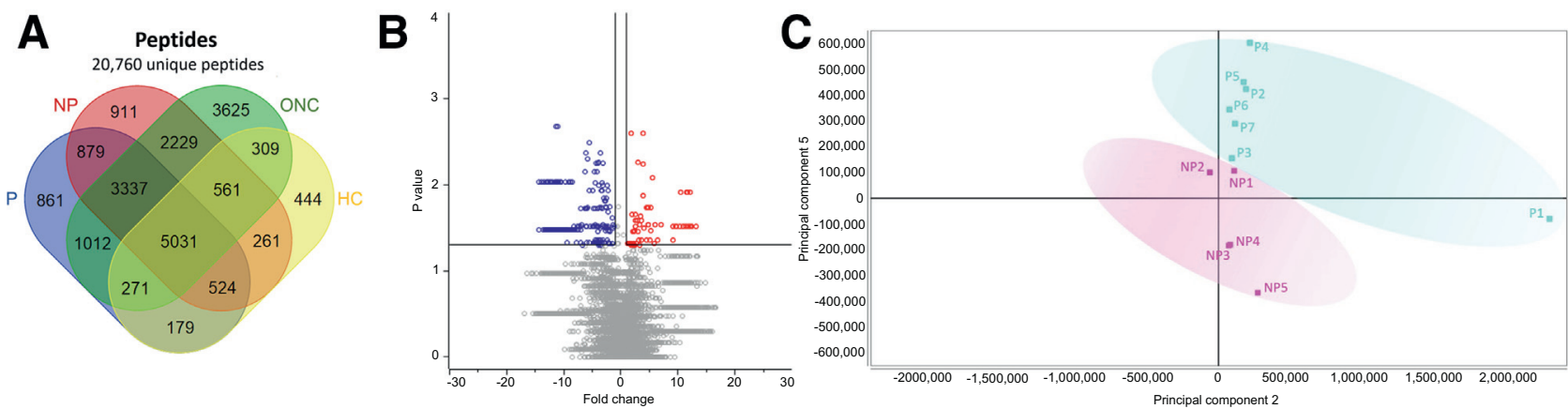

\section{D}



Figure 1 Characterization of the urinary peptidome in progressive and nonprogressive early-stage clear cell tumors. A: Venn diagram of the 20,760 nonredundant endogenous peptides detected in healthy controls $(\mathrm{HC})$, renal oncocytoma $(0 \mathrm{NC} ; \leq 4 \mathrm{~cm})$, progressive $(\mathrm{P})$ and nonprogressive $(\mathrm{NP})$ clear cell renal cell carcinoma (cCRCC) urine samples by shotgun peptidomic analysis. B: Volcano plot illustrating significantly dysregulated peptides between progressive and nonprogressive early-stage ccRCC tumors $(P<0.05)$. C: Principal component analysis of progressive and nonprogressive clear cell tumors based on the intensity values of differentially expressed endogenous peptides $(P<0.05)$. D: Unsupervised hierarchical clustering of differentially expressed endogenous peptides between progressive and nonprogressive clear cell tumors [352 endogenous peptides; $P<0.05$ ( $U$-test)]. Data are expressed as means \pm SD. $n=5(\mathrm{NP}) ; n=6(\mathrm{ONC}) ; n=7(\mathrm{P})$. 
endogenous peptides that were differentially expressed between healthy controls and ccRCC-SRMs (pT1a; $\leq 4 \mathrm{~cm}$ ). Peptide DEPPQSPWDRVKDLATV was significantly elevated in early-stage ccRCC (2.22-fold change, $P<0.001)$ and showed the most significant discriminatory utility (AUC, $0.71 ; 95 \%$ CI, 0.58-0.84; $P=0.0074$ ) (Figure 2, A and B). LQKPGQSPQLL was significantly elevated in early-stage ccRCC (2.30-fold change, $P=0.018$ ), and was able to discriminate between the two groups (AUC, $0.70 ; 95 \% \mathrm{CI}, 0.54-0.83 ; P=0.0175$ ) (Figure 2, C and D). ACDDYRLCERY was significantly up-regulated in early-stage ccRCC relative to healthy controls (3.04-fold change, $P=0.0017$ ), and showed significant discriminatory utility (AUC, $0.70 ; 95 \%$ CI, 0.56-0.82; $P=0.0156$ ) (Figure 2, E and F). Peptides DFINDATDVNDALGYVTRF $(P=0.022)$, FINDATDVNDALGYVTRF $(P=0.039)$, INEQWLLTTAKNL $(P=0.009)$, LDTYPNDETTERVFPYISV $(P=0.032)$, INDATDVNDALGYVTRF $(P=0.016)$, and YSNEKPFTGFE $(P=0.046)$ displayed significantly elevated expression in ccRCC-SRMs relative to healthy controls (Figure 2, G-L). In addition, peptides
DFINDATDVNDALGYVTRF (AUC, 0.63; 95\% CI, 0.49-0.78), FINDATDVNDALGYVTRF (AUC, 0.63; 95\% CI, 0.48-0.78), INEQWLLTTAKNL (AUC, 0.64; 95\% CI, 0.5-0.78), LDTYPNDETTERVFPYISV (AUC, $0.68 ; 95 \%$ CI, 0.53-0.82), INDATDVNDALGYVTRF (AUC, $0.65 ; 95 \% \mathrm{CI}, 0.51-0.79$ ), and YSNEKPFTGFE (AUC, 0.66; 95\% CI, 0.52-0.81) showed significant discriminatory utility $(P<0.05)$ (data not shown).

The prognostic utility of peptides displaying significantly elevated expression in early-stage clear cell tumors relative to healthy controls was assessed in our patient cohort. Kaplan-Meier analysis showed that patients with elevated YSNEKPFTGFE expression had significantly shorter overall survival [hazard ratio (HR), 7.76; 95\% CI, 1.82-33.04; $P=0.0012$ ] compared to patients with lower YSNEKPFTGFE expression levels (Supplemental Figure S5). Peptides DEPPQSPWDRVKDLATV, LQKPGQSPQLL, ACDDYRLCERY, DFINDATDVNDALGYVTRF, FINDATDVNDALGYVTRF, INEQWLLTTAKNL, LDTYPNDETTERVFPYISV, and INDATDVNDALGYVTRF were not associated with survival.
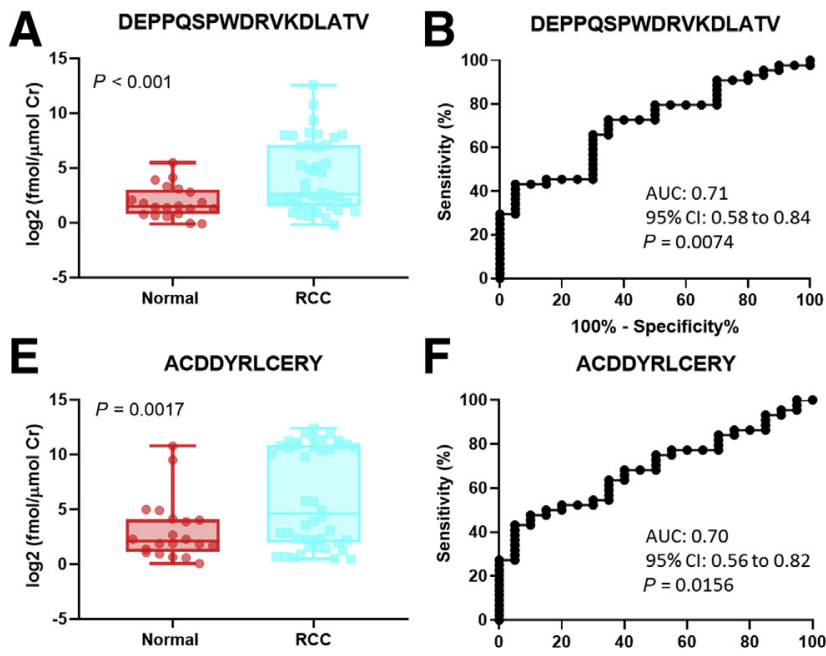

\section{$\mathbf{F}$}
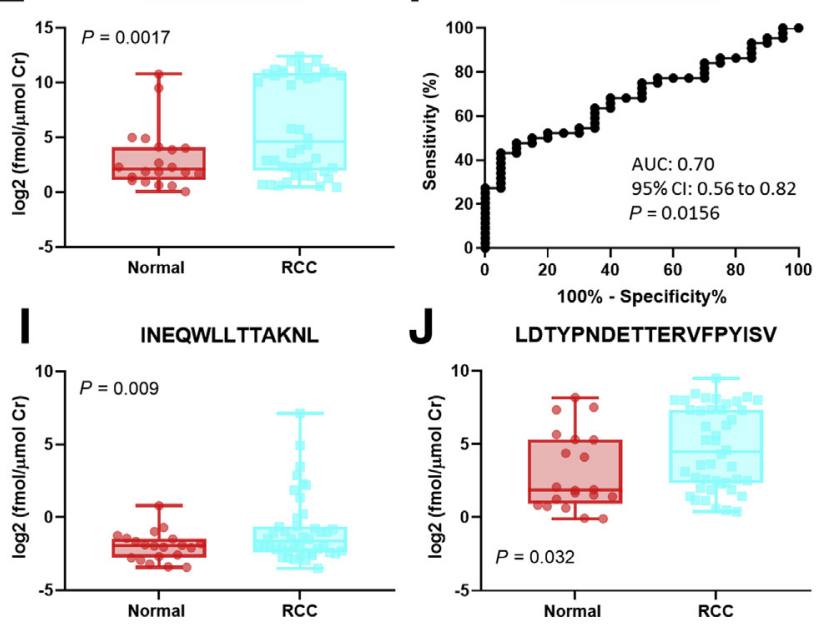
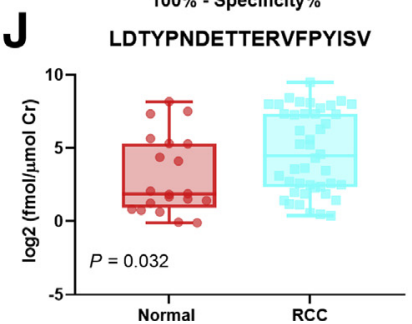
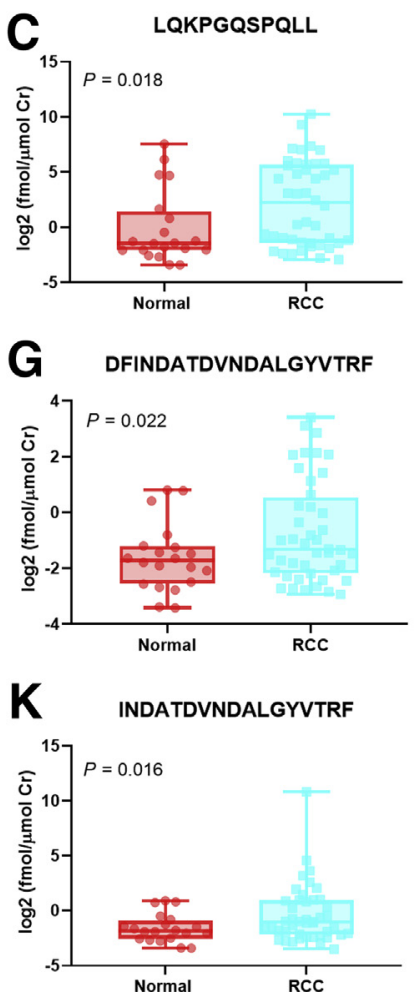

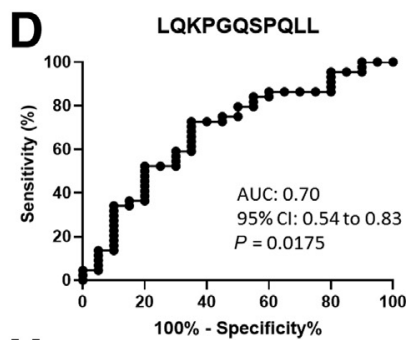

H

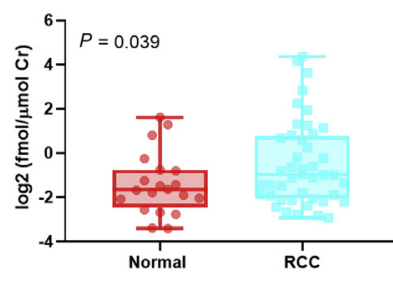

$\mathbf{L}$

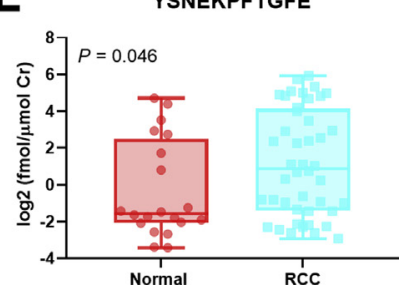

Figure 2 Relative quantification of endogenous urinary peptides in early-stage clear cell tumors and healthy controls by parallel-reaction monitoring. A: Relative expression of DEPPQSPWDRVKDLATV in early-stage clear cell tumors [clear cell renal cell carcinoma (RCC)] and healthy controls (Normal). B: Receiver operating characteristic (ROC) curve showing the discriminatory utility of DEPPQSPWDRVKDLATV. C: Relative expression of LQKPGQSPQLL in early-stage clear cell tumors and healthy controls. D: ROC curve showing the discriminatory utility of LQKPGQSPQLL. E: Relative expression of ACDDYRLCERY in early-stage clear cell tumors and healthy controls. F: ROC curve showing the discriminatory utility of ACDDYRLCERY. G-L: Relative expression of DFINDATDVNDALGYVTRF (G), FINDATDVNDALGYVTRF (H), INEQWLLTTAKNL (I), LDTYPNDETTERVFPYISV (J), INDATDVNDALGYVTRF (K), and YSNEKPFTGFE (L) in early-stage clear cell tumors and healthy controls. Data are expressed as log2-transformed fmol/ $\mu \mathrm{mol}$ of urine creatinine ( $\mathrm{Cr}$ ). Box plots are expressed as means (horizontal lines), 10 to 90 percentile (boxes), and extent of data (whiskers). $n=20$ (normal); $n=44$ (RCC). 
Use of Endogenous Peptides in Distinguishing Renal Oncocytoma $\leq 4 \mathrm{~cm}$ from Early-Stage ccRCC

Univariate analysis identified three endogenous peptides that were differentially expressed between renal oncocytoma $(\leq 4 \mathrm{~cm})$ and ccRCC-SRMs (pTla; $\leq 4 \mathrm{~cm})$. NVINGGSHAGNKLAMQEF was significantly upregulated in ccRCC-SRMs (2.83-fold change, $P=0.017$ ) (Figure 3A) and showed significant discriminatory utility (AUC, 0.62; 95\% CI, 0.49-0.75; $P=0.01$ ). Peptides VNVDEVGGEALGRL (2.0-fold change, $P=0.025)$ and VVAGVANALAHKYH (2.11-fold change, $P=0.019$ ) also displayed significantly elevated expression in earlystage ccRCC relative to renal oncocytoma $(\leq 4 \mathrm{~cm})$ (Figure 3, B and C). In addition, peptides VNVDEVGGEALGRL (AUC, 0.63 ; 95\% CI, 0.5-0.76) and VVAGVANALAHKYH (AUC, 0.62; 95\% CI, 0.49-0.76) showed significant discriminatory utility $(P<0.05)$.

The prognostic utility of VNVDEVGGEALGRL, VVAGVANALAHKYH, and NVINGGSHAGNKLAMQEF was assessed in our patient cohort. Kaplan-Meier analysis showed that patients with elevated NVINGGSHAGNKLAMQEF expression had significantly shorter overall survival (HR, 3.9; 95\% CI, 1.19-12.82; $P=0.016$ ) compared to patients with lower NVINGGSHAGNKLAMQEF expression levels (Figure 3D). Since NVINGGSHAGNKLAMQEF is a fragment of $\alpha$-enolase (ENO1), the prognostic utility of ENO1 was assessed in an independent cohort of ccRCC (pT1) patients from The Cancer Genome Atlas. Patients with higher ENO1-expressing tumors had significantly longer overall survival (HR, $0.23 ; 95 \%$ CI, $0.09-0.59 ; P=0.0008$ ) compared to patients with lower ENO1-expressing tumors (Figure $3 \mathrm{E}$ ). This seemingly contradictory finding may have been due to increased proteolysis of ENO1 by an unknown protease, which would explain, in part, the observed increase in NVINGGSHAGNKLAMQEF levels in early-stage ccRCC.

Use of Endogenous Urinary Peptides in Distinguishing Progressive from Nonprogressive Early-Stage cCRCC

The utility of endogenous urinary peptides in distinguishing between progressive and nonprogressive ccRCC-SRMs (pT1a; $\leq 4 \quad \mathrm{~cm})$ was next examined. Peptide SHTSDSDVPSGVTEVVVKL was significantly elevated in progressive early-stage clear cell tumors (2.22-fold change, $P=0.0032)$ and showed the most significant discriminatory utility (AUC, $0.76 ; 95 \% \mathrm{CI}, 0.62-0.90 ; P=0.0027$ )
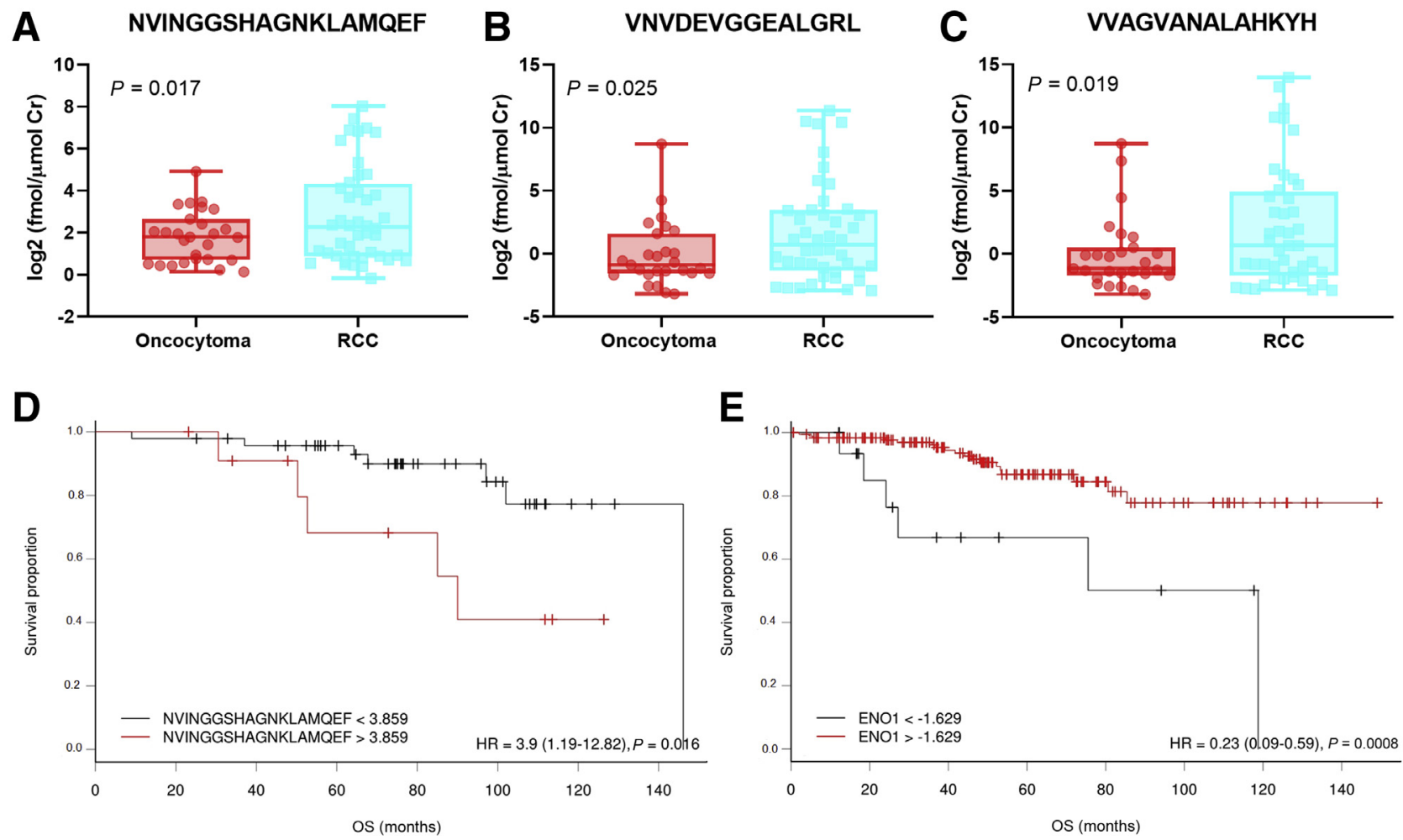

Figure 3 Relative quantification of endogenous urinary peptides in early-stage clear cell tumors and renal oncocytoma $(\leq 4 \mathrm{~cm})$ by parallel-reaction monitoring. A-C: Relative expression of NVINGGSHAGNKLAMQEF (A), VNVDEVGGEALGRL (B), and VVAGVANALAHKYH (C) in early-stage clear cell tumors [clear cell renal cell carcinoma (ccRCC)] and renal oncocytoma (Oncocytoma). D: Kaplan-Meier survival curves showing the association between relative NVINGGSHAGNKLAMQEF expression and overall survival (OS) in our patient sample cohort. E: Kaplan-Meier survival curves showing the association between EN01 expression and OS in an independent cohort of ccRCC (pT1) patients from The Cancer Genome Atlas. Data are expressed as log2-transformed fmol/ $\mu$ mol of urine creatinine. Box plots are expressed as means (horizontal lines), 10 to 90 percentile (boxes), and extent of data (whiskers). $n=27$ oncocytoma; $n=44$ CCRCC. 
(Figure 4, A and B). IVDNNILFLGKVNRP was significantly up-regulated in progressive early-stage clear cell tumors (2.66-fold change, $P=0.014$ ) and was useful in discriminating between the two groups (AUC, 0.70; 95\% CI, 0.53-0.84; $P=0.0297$ ) (Figure 4, C and D).

\section{Prognostic Value of Peptide SHTSDSDVPSGVTEVVVKL in Early-Stage Clear Cell RCC}

Since SHTSDSDVPSGVTEVVVKL displayed the most significant discriminatory utility in distinguishing progressive from nonprogressive tumors, the prognostic utility of this peptide was assessed in our patient population. The association between SHTSDSDVPSGVTEVVVKL expression and different clinicopathologic characteristics was tested. As a binary variable, there was no significant association between SHTSDSDVPSGVTEVVVKL expression and sex, age, laterality, tumor size, or tumor grade (Table 1), suggesting that SHTSDSDVPSGVTEVVVKL is an independent marker of poor prognosis in early-stage tumors.

The prognostic utility of SHTSDSDVPSGVTEVVVKL and IVDNNILFLGKVNRP expression was also assessed in our patient cohort. Kaplan-Meier analysis showed that patients with elevated SHTSDSDVPSGVTEVVVKL expression had significantly shorter overall survival (HR, 4.13; 95\% CI, 1.09-15.65; $P=0.024)$ relative to patients with lower SHTSDSDVPSGVTEVVVKL levels (Figure 4E). Patients with elevated IVDNNILFLGKVNRP expression also displayed worse overall survival relative to patients with lower IVDNNILFLGKVNRP expression levels; however, the difference was not statistically significant (HR, 2.29; 95\% CI, $0.61-8.65 ; P=0.21$ ) (Figure 4F). Since SHTSDSDVPSGVTEVVVKL is a fragment of CLU, the prognostic utility of CLU was assessed in an independent cohort of ccRCC (pT1) patients from The Cancer Genome Atlas. Patients with higher CLU-expressing tumors had significantly longer overall survival (HR, 0.4; 95\% CI, 0.17-0.94; $P=0.03$ ) compared to patients with lower CLU-expressing tumors (Figure 4G). This seemingly contradictory finding may have been due to increased proteolysis of CLU by an unknown protease, which would explain, in part, the observed increase in SHTSDSDVPSGVTEVVVKL levels in progressive tumors.

\section{Discussion}

RCC is often detected incidentally as an SRM (pT1a, $\leq 4$ $\mathrm{cm}) .{ }^{22}$ Current imaging methods are not useful in reliably discriminating oncocytoma $(\leq 4 \mathrm{~cm})$ from early-stage ccRCC. $^{23,24}$ Moreover, there are no available markers that can be used for predicting progression. The development of noninvasive biomarkers for early-stage tumors could significantly improve clinical management and reduce overtreatment.

Peptidomics has emerged as a promising tool for the discovery of noninvasive cancer biomarkers. ${ }^{10}$ Endogenous urinary peptides represent useful biomarkers as they are
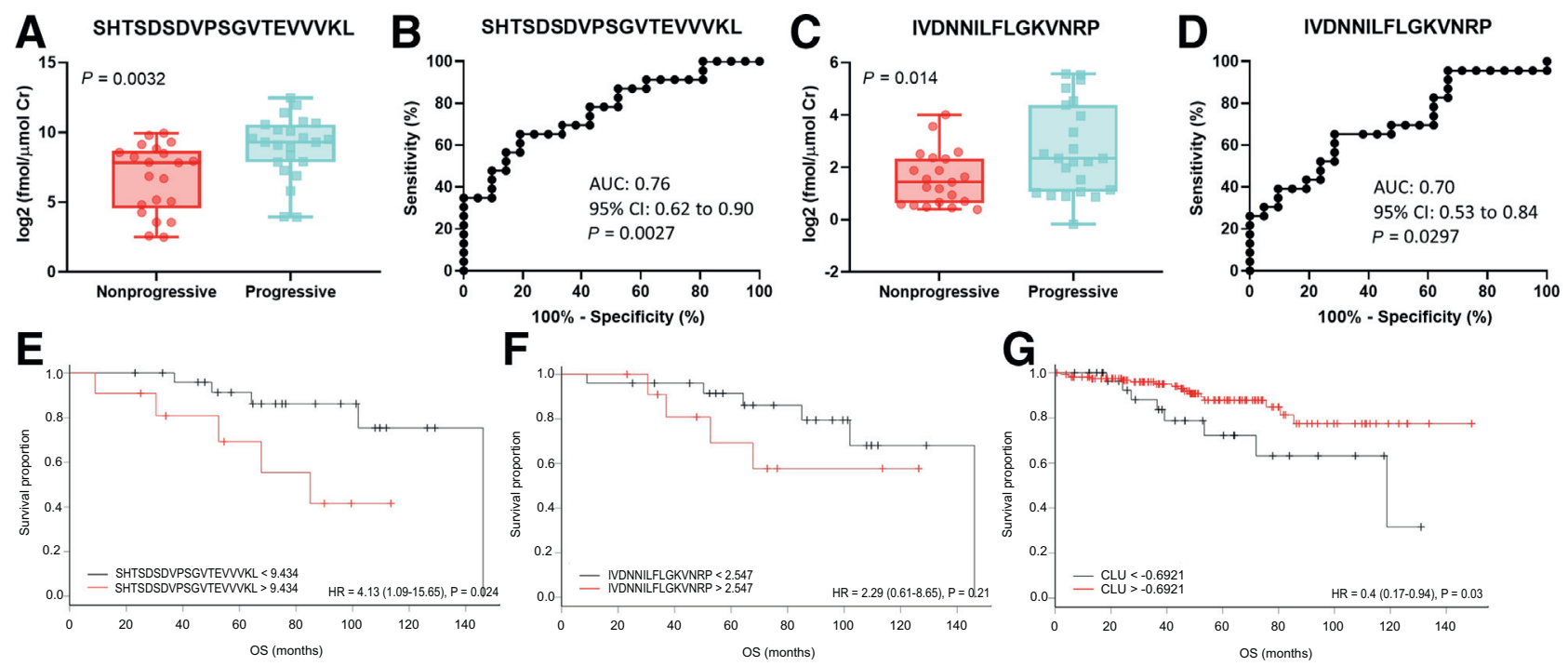

Figure 4 Relative quantification of endogenous urinary peptides in progressive and nonprogressive early-stage clear cell tumors by parallel-reaction monitoring. A: Relative expression of SHTSDSDVPSGVTEVVVKL in progressive and nonprogressive early-stage clear cell tumors. B: Receiver operating characteristic (ROC) curve showing the discriminatory utility of SHTSDSDVPSGVTEVVVKL. C: Relative expression of IVDNNILFLGKVNRP in progressive and nonprogressive early-stage tumors. D: ROC curve showing the discriminatory utility of IVDNNILFLGKVNRP. E and F: Kaplan-Meier survival curves showing the association between relative SHTSDSDVPSGVTEVVVKL (E) and IVDNNILFLGKVNRP (F) expression and overall survival (OS) in our patient sample cohort. G: Kaplan-Meier survival curves showing the association between clustering (CLU) expression and OS in an independent cohort of clear cell renal cell carcinoma (pT1) patients from The Cancer Genome Atlas. Relative expression data are expressed as log2-transformed fmol/ $\mu$ mol of urine creatinine. Box plots are expressed as means (horizontal lines), 10 to 90 percentile (boxes), and extent of data (whiskers). $n=21$ (nonprogressive); $n=23$ (progressive); $n=44$ (patient sample cohort). 
Table 1 Associations between SHTSDSDVPSGVTEVVVKL Status and Clinicopathologic Variables in ccRCC

\begin{tabular}{|c|c|c|c|c|}
\hline \multirow[b]{2}{*}{ Variable } & \multirow[b]{2}{*}{ Total, $n$} & \multicolumn{2}{|l|}{ Patients, $n(\%)$} & \multirow[b]{2}{*}{$P$ value } \\
\hline & & Low SHTSDSDVPSGVTEVVVKL & High SHTSDSDVPSGVTEVVVKL & \\
\hline Sex & & & & $0.1159 *$ \\
\hline Male & 28 & $11(39.3)$ & $17(60.7)$ & \\
\hline Female & 16 & $11(68.7)$ & $5(31.3)$ & \\
\hline Age, years & & & & $0.7635^{*}$ \\
\hline$\leq 83$ & 22 & $10(45.45)$ & $12(54.55)$ & \\
\hline$>83$ & 22 & $12(54.55)$ & $10(45.45)$ & \\
\hline Laterality & & & & $0.3292^{\dagger}$ \\
\hline Right & 24 & $12(50.0)$ & $12(50.0)$ & \\
\hline Left & 18 & $8(44.4)$ & $10(55.6)$ & \\
\hline Unknown & 2 & $2(100)$ & 0 & \\
\hline Tumor size & & & & $0.1040^{\dagger}$ \\
\hline$\leq 2.5 \mathrm{~cm}$ & 22 & $13(59.1)$ & $9(40.9)$ & \\
\hline$>2.5 \mathrm{~cm}$ & 20 & $7(35.0)$ & $13(65.0)$ & \\
\hline Unknown & 2 & $2(100)$ & 0 & \\
\hline Fuhrman grade & & & & $0.4999^{\dagger}$ \\
\hline $\mathrm{I}$ & 20 & $8(40.0)$ & $12(60.0)$ & \\
\hline II & 15 & $8(53.3)$ & $7(46.7)$ & \\
\hline III & 1 & $1(100)$ & 0 & \\
\hline $\mathrm{IV}^{\ddagger}$ & 0 & 0 & 0 & \\
\hline Unknown & 8 & $5(62.5)$ & $3(37.5)$ & \\
\hline
\end{tabular}

*Fisher exact test.

${ }^{\dagger}$ Pearson $\chi^{2}$ test.

${ }^{\ddagger}$ Not included in statistical test.

ccRCC, clear cell renal cell carcinoma.

both abundant and easily accessible. ${ }^{25}$ Here, the utility of urinary peptides in discriminating early-stage ccRCC (pT1a; $\leq 4 \mathrm{~cm})$ from healthy controls and renal oncocytoma $(\leq 4$ $\mathrm{cm}$ ) was investigated by label-free LC-MS/MS and targeted PRM. Additionally, it was examined whether urinary peptides could be used for predicting aggressive behavior in early-stage tumors. To our knowledge, this study is the first to investigate urinary peptides as biomarkers of aggressive behavior in pT1a tumors.

Nine urinary peptides were identified as useful in distinguishing early-stage clear cell tumors $(\mathrm{pT} 1 \mathrm{a} ; \leq 4 \mathrm{~cm})$ from healthy controls. Peptide DEPPQSPWDRVKDLATV displayed the best diagnostic performance. Peptides identified on univariate analysis originate from proteins previously linked to RCC, including apolipoprotein A-I (DEPPQSPWDRVKDLATV), haptoglobin (INEQWLLTTAKNL), lectin mannose-binding 2 (LDTYPNDETTERVFPYISV), and mannan-binding lectin serine protease 2 (YSNEKPFTGFE). ${ }^{26-28}$ A study by Sandim et $\mathrm{al}^{26}$ found that urine levels of apolipoprotein A-I and haptoglobin were significantly elevated in ccRCC relative to healthy controls. That finding is in line with those from the present study, as apolipoprotein A-I and haptoglobin protein fragments DEPPQSPWDRVKDLATV and INEQWLLTTAKNL were shown to be significantly elevated in early-stage tumors compared to healthy controls. Another study found that plasma levels of lectin mannose-binding 2 were significantly higher in RCC patients compared to healthy controls. ${ }^{27}$ This aligns with the present findings as LDTYPNDETTERVFPYISV, a lectin mannose-binding 2 protein fragment, was shown to be significantly elevated in early-stage tumors compared to healthy controls. Consistent with the present findings, Pieper et $\mathrm{al}^{28}$ showed that urine levels of mannanbinding lectin serine protease 2 decreased significantly after surgery in patients with RCC. In the present study, YSNEKPFTGFE, a mannan-binding lectin serine protease 2 protein fragment, displayed significantly elevated expression in early-stage tumors compared to healthy controls.

In addition, NVINGGSHAGNKLAMQEF, VNVDEVGGEALGRL, and VVAGVANALAHKYH could significantly distinguish early-stage ccRCC (pT1a; $\leq 4 \mathrm{~cm}$ ) from renal oncocytoma $(\leq 4 \mathrm{~cm})$. Interestingly, patients with elevated NVINGGSHAGNKLAMQEF expression displayed significantly shorter overall survival compared to patients with low expression. Peptides identified on univariate analysis originate from proteins previously reported in RCC, including ENO1 (NVINGGSHAGNKLAMQEF). ${ }^{27,29-31}$ Several studies have shown that ENO1 is significantly elevated in RCC. ${ }^{27,29,30}$ Our present findings of elevated NVINGGSHAGNKLAMQEF levels in urine biopsy specimens from patients with early-stage clear cell tumors align with this, suggesting that NVINGGSHAGNKLAMQEF may be an early diagnostic marker. Recently, low ENO1 expression has been found to be associated with poor prognosis in ccRCC patients. ${ }^{31}$ This finding aligns with those in the present study, as patients with low ENO1 expression had significantly 
shorter overall survival. Interestingly, the opposite trend was observed for NVINGGSHAGNKLAMQEF expression, such that patients with low NVINGGSHAGNKLAMQEF expression had significantly longer overall survival. We speculate that differences in survival may have been due to proteolytic processing of ENO1; however, this concept requires further investigation.

Additionally, two early markers of ccRCC progression were identified. SHTSDSDVPSGVTEVVVKL could be used for significantly distinguishing progressive from nonprogressive tumors (pT1a; $\leq 4 \mathrm{~cm}$ ), and elevated SHTSDSDVPSGVTEVVVKL levels were associated with significantly shorter overall survival. Peptides identified on univariate analysis originate from proteins previously reported in RCC, including CLU (SHTSDSDVPSGVTEVVVKL) and plasma serine protease inhibitor (SERPINA5; IVDNNILFLGKVNRP). ${ }^{32-39}$ Several studies have shown that CLU is elevated in RCC relative to normal renal tissues. ${ }^{32,33}$ In the present study, patients with low CLU expression had significantly shorter overall survival. The opposite trend was observed for SHTSDSDVPSGVTEVVVKL expression, such that patients with low SHTSDSDVPSGVTEVVVKL expression had significantly longer overall survival. The differences in survival may have been due to proteolytic processing of CLU; however, this idea requires further investigation. Several reports have shown that SERPINA5 is significantly down-regulated in ccRCC versus adjacent normal tissue. $^{34-37}$ Moreover, low SERPINA5 expression was previously found to have been associated with poor survival in RCC. ${ }^{38,39}$ Here, the opposite trend was observed for IVDNNILFLGKVNRP, a SERPINA5 peptide fragment, such that patients with low IVDNNILFLGKVNRP expression had longer overall survival; however, this trend did not reach statistical significance. Again, this finding may have been a result of proteolytic processing of SERPINA5, although this hypothesis requires further study.

Interestingly, peptide DDPDAPLQPVTPLQLFEGRRN, which has been found to be significantly up-regulated in progressive tumors in the discovery phase, was previously identified. Huang et al ${ }^{15}$ showed that serum levels of peptide DDPDAPLQPVTPLQLFEGRRN were significantly upregulated in RCC patients relative to controls. Although peptide DDPDAPLQPVTPLQLFEGRRN was not selected for further investigation in the present study, the data suggest that this peptide may be an early disease marker.

This study had some limitations. First, non-clear cell tumors, such as papillary and chromophobe RCC, were not considered. Instead ccRCC was studied as it is the most predominant subtype of RCC. ${ }^{3}$ Other nontumor renal pathologies, such as angiomyolipoma, cystic disease, fibrosis, and glomerulosclerosis, were also not considered. However, recent evidence suggests that angiomyolipoma can be safely diagnosed by imaging. ${ }^{40}$ The use of histology as the gold standard of SRM diagnosis represents another limitation. Pathologists frequently struggle to accurately classify incidentally detected SRMs (pT1a; $\leq 4 \mathrm{~cm}$ ); however, recent evidence suggests that renal biopsy is a safe and accurate technique for diagnosis. ${ }^{41}$

In the present study, the utility of noninvasive endogenous peptides as potential diagnostic and prognostic markers in early-stage renal tumors (SRMs; pT1a, $\leq 4 \mathrm{~cm}$ ) was demonstrated. Although the performance of the peptide markers is not ideal, they may help to assign patients to appropriate management programs. In patients with clinically indolent tumors, active surveillance may be considered as an option alternative to immediate surgical intervention.

\section{Supplemental Data}

Supplemental material for this article can be found at https://doi.org/10.1016/j.ajpath.2019.08.015.

\section{References}

1. Butz H, Szabo PM, Khella HW, Nofech-Mozes R, Patocs A Yousef GM: miRNA-target network reveals miR-124as a key miRNA contributing to clear cell renal cell carcinoma aggressive behaviour by targeting CAV1 and FLOT1. Oncotarget 2015, 6: $12543-12557$

2. Chinello C, Cazzaniga M, De Sio G, Smith AJ, Gianazza E, Grasso A, Rocco F, Signorini S, Grasso M, Bosari S, Zoppis I, Dakna M, van der Burgt YE, Mauri G, Magni F: Urinary signatures of renal cell carcinoma investigated by peptidomic approaches. PLoS One 2014, 9:e106684

3. Shuch B, Amin A, Armstrong AJ, Eble JN, Ficarra V, LopezBeltran A, Martignoni G, Rini BI, Kutikov A: Understanding pathologic variants of renal cell carcinoma: distilling therapeutic opportunities from biologic complexity. Eur Urol 2015, 67:85-97

4. Jewett MA, Rendon R, Lacombe L, Karakiewicz PI, Tanguay S, Kassouf W, Leveridge M, Cagiannos I, Kapoor A, Pautler S, Drachtenberg D, Moore R, Gleave M, Evans A, Haider M, Finelli A: Canadian guidelines for the management of small renal masses (SRM). Can Urol Assoc J 2015, 9:160-163

5. Jewett MA, Richard PO, Finelli A: Management of small renal mass: an opportunity to address a growing problem in early stage kidney cancer. Eur Urol 2015, 68:416-417

6. Cheung DC, Finelli A: Active surveillance in small renal masses in the elderly: a literature review. Eur Urol Focus 2017, 3:340-351

7. Frantzi M, Metzger J, Banks RE, Husi H, Klein J, Dakna M, Mullen W, Cartledge JJ, Schanstra JP, Brand K, Kuczyk MA, Mischak H, Vlahou A, Theodorescu D, Merseburger AS: Discovery and validation of urinary biomarkers for detection of renal cell carcinoma. J Proteomics 2014, 98:44-58

8. Jewett MA, Mattar K, Basiuk J, Morash CG, Pautler SE, Siemens DR, Tanguay S, Rendon RA, Gleave ME, Drachenberg DE, Chow R, Chung H, Chin JL, Fleshner NE, Evans AJ, Gallie BL, Haider MA, Kachura JR, Kurban G, Fernandes K, Finelli A: Active surveillance of small renal masses: progression patterns of early stage kidney cancer. Eur Urol 2011, 60:39-44

9. Di Meo A, Saleeb R, Wala SJ, Khella HW, Ding Q, Zhai H, Krishan K, Krizova A, Gabril M, Evans A, Brimo F, Pasic MD, Finelli A, Diamandis EP, Yousef GM: A miRNA-based classification of renal cell carcinoma subtypes by PCR and in situ hybridization. Oncotarget 2018, 9:2092-2104 
10. Di Meo A, Bartlett J, Cheng Y, Pasic MD, Yousef GM: Liquid biopsy: a step forward towards precision medicine in urologic malignancies. Mol Cancer 2017, 16:80

11. Pisitkun T, Shen RF, Knepper MA: Identification and proteomic profiling of exosomes in human urine. Proc Natl Acad Sci U S A 2004, 101:13368-13373

12. Di Meo A, Batruch I, Yousef AG, Pasic MD, Diamandis EP, Yousef GM: An integrated proteomic and peptidomic assessment of the normal human urinome. Clin Chem Lab Med 2017, 55:237-247

13. Di Meo A, Pasic MD, Yousef GM: Proteomics and peptidomics: moving toward precision medicine in urological malignancies. Oncotarget 2016, 7:52460-52474

14. Chinello C, Cazzaniga M, De Sio G, Smith AJ, Grasso A, Rocco B, Signorini S, Grasso M, Bosari S, Zoppis I, Mauri G, Magni F: Tumor size, stage and grade alterations of urinary peptidome in RCC. J Transl Med 2015, 13:332

15. Huang Z, Zhang S, Hang W, Chen Y, Zheng J, Li W, Xing J, Zhang J, Zhu E, Yan X: Liquid chromatography-mass spectrometry based serum peptidomic approach for renal clear cell carcinoma diagnosis. J Pharm Biomed Anal 2014, 100:175-183

16. Cox J, Mann M: MaxQuant enables high peptide identification rates, individualized p.p.b.-range mass accuracies and proteome-wide protein quantification. Nat Biotechnol 2008, 26:1367-1372

17. Cox J, Hubner NC, Mann M: How much peptide sequence information is contained in ion trap tandem mass spectra? J Am Soc Mass Spectrom 2008, 19:1813-1820

18. Begcevic I, Brinc D, Brown M, Martinez-Morillo E, Goldhardt O, Grimmer T, Magdolen V, Batruch I, Diamandis EP: Brain-related proteins as potential CSF biomarkers of Alzheimer's disease: a targeted mass spectrometry approach. J Proteomics 2018, 182:12-20

19. Zhang Z: Data management by using R: big data clinical research series. Ann Transl Med 2015, 3:303

20. Tyanova S, Temu T, Sinitcyn P, Carlson A, Hein MY, Geiger T, Mann M, Cox J: The Perseus computational platform for comprehensive analysis of (prote)omics data 2016, 13:731-740

21. Reich M, Tabor T Liefeld T, Thorvaldsdóttir H, Hill B, Tamayo P, Mesirov JP: The GenePattern notebook environment. Cell Syst 2017, 5:149-151.e1

22. Kane CJ, Mallin K, Ritchey J, Cooperberg MR, Carroll PR: Renal cell cancer stage migration: analysis of the National Cancer Data Base. Cancer 2008, 113:78-83

23. Richard PO, Jewett MA, Tanguay S, Saarela O, Liu ZA, Pouliot F, Kapoor A, Rendon R, Finelli A: Safety, reliability and accuracy of small renal tumour biopsies: results from a multi-institution registry. BJU Int 2017, 119:543-549

24. Ambani SN, Wolf JS Jr: Renal mass biopsy for the small renal mass. Urol Oncol 2018, 36:4-7

25. Sigdel TK, Nicora CD, Hsieh SC, Dai H, Qian WJ, Camp DG 2nd, Sarwal MM: Optimization for peptide sample preparation for urine peptidomics. Clin Proteomics 2014, 11:7

26. Sandim V, Pereira Dde A, Kalume DE, Oliveira-Carvalho AL, Ornellas AA, Soares MR, Alves G, Zingali RB: Proteomic analysis reveals differentially secreted proteins in the urine from patients with clear cell renal cell carcinoma. Urol Oncol 2016, 34:5.e11-5.e25

27. Kaneko N, Gotoh A, Okamura N, Matsuo E, Terao S, Watanabe M, Yamada Y, Hamami G, Nakamura T, Ikekita M, Okumura K, Nishimura O: Potential tumor markers of renal cell carcinoma: alphaenolase for postoperative follow up, and galectin- 1 and galectin- 3 for primary detection. Int J Urol 2013, 20:530-535
28. Pieper R, Gatlin CL, McGrath AM, Makusky AJ, Mondal M, Seonarain M, Field E, Schatz CR, Estock MA, Ahmed N, Anderson NG, Steiner S: Characterization of the human urinary proteome: a method for high-resolution display of urinary proteins on two-dimensional electrophoresis gels with a yield of nearly 1400 distinct protein spots. Proteomics 2004, 4:1159-1174

29. White NM, Masui O, Desouza LV, Krakovska O, Metias S, Romaschin AD, Honey RJ, Stewart R, Pace K, Lee J, Jewett MA, Bjarnason GA, Siu KW, Yousef GM: Quantitative proteomic analysis reveals potential diagnostic markers and pathways involved in pathogenesis of renal cell carcinoma. Oncotarget 2014, 5:506-518

30. Zhao Q, Kun D, Hong B, Deng X, Guo S, Tang X, Yang Y, Gong K, Li Q, Ye L, Jiang WG, Zhang N: Identification of novel proteins interacting with vascular endothelial growth inhibitor 174 in renal cell carcinoma. Anticancer Res 2017, 37:4379-4388

31. White-Al Habeeb NM, Di Meo A, Scorilas A, Rotondo F, Masui O, Seivwright A, Gabril M, Girgis AH, Jewett MA, Yousef GM: Alphaenolase is a potential prognostic marker in clear cell renal cell carcinoma. Clin Exp Metastasis 2015, 32:531-541

32. Zellweger T, Miyake H, July LV, Akbari M, Kiyama S, Gleave ME Chemosensitization of human renal cell cancer using antisense oligonucleotides targeting the antiapoptotic gene clusterin. Neoplasia 2001, 3:360-367

33. Kurahashi T, Muramaki M, Yamanaka K, Hara I, Miyake H: Expression of the secreted form of clusterin protein in renal cell carcinoma as a predictor of disease extension. BJU Int 2005, 96:895-899

34. Zaravinos A, Pieri M, Mourmouras N, Anastasiadou N, Zouvani I, Delakas D, Deltas C: Altered metabolic pathways in clear cell renal cell carcinoma: a meta-analysis and validation study focused on the deregulated genes and their associated networks. Oncoscience 2014 , $1: 117-131$

35. Zodro E, Jaroszewski M, Ida A, Wrzesinski T, Kwias Z, Bluyssen H, Wesoly J: FUT11 as a potential biomarker of clear cell renal cell carcinoma progression based on meta-analysis of gene expression data. Tumour Biol 2014, 35:2607-2617

36. Wozniak MB, Le Calvez-Kelm F, Abedi-Ardekani B, Byrnes G, Durand G, Carreira C, Michelon J, Janout V, Holcatova I, Foretova L, Brisuda A, Lesueur F, McKay J, Brennan P, Scelo G: Integrative genome-wide gene expression profiling of clear cell renal cell carcinoma in Czech Republic and in the United States. PLoS One 2013, 8: e57886

37. Wakita T, Hayashi T, Nishioka J, Tamaru H, Akita N, Asanuma K, Kamada H, Gabazza EC, Ido M, Kawamura J, Suzuki K: Regulation of carcinoma cell invasion by protein $\mathrm{C}$ inhibitor whose expression is decreased in renal cell carcinoma. Int J Cancer 2004, 108:516-523

38. Takahashi M, Rhodes DR, Furge KA, Kanayama H, Kagawa S, Haab BB, Teh BT: Gene expression profiling of clear cell renal cell carcinoma: gene identification and prognostic classification. Proc Natl Acad Sci U S A 2001, 98:9754-9759

39. Lenburg ME, Liou LS, Gerry NP, Frampton GM, Cohen HT, Christman MF: Previously unidentified changes in renal cell carcinoma gene expression identified by parametric analysis of microarray data. BMC Cancer 2003, 3:31

40. Thiravit S, Teerasamit W, Thiravit P: The different faces of renal angiomyolipomas on radiologic imaging: a pictorial review. Br J Radiol 2018, 91:20170533

41. Herrera-Caceres JO, Finelli A, Jewett MAS: Renal tumor biopsy: indicators, technique, safety, accuracy results, and impact on treatment decision management. World J Urol 2019, 37:437-443 